\title{
Transferability and specialization: analyzing STEM students' perspectives of problem-solving
}

Vina Macias, Jacob Poirier, Benjamin M Zwickl, and Susan Rothwell

School of Physics and Astronomy, Rochester Institute of Technology,

1 Lomb Memorial Drive, Rochester, New York, 14623

Problem-solving is emphasized in introductory physics courses to equip students with tools that hopefully transfer to their future lives and careers. The EMPOWER project interviewed undergraduate STEM majors from computing, manufacturing, nursing, energy, and physics about their perspectives on problem-solving. Sixty-nine student focus groups were conducted, totaling 239 students. All students emphasized the importance of problem-solving in their discipline of study, but no single practice of problem-solving was applicable for all students. Most problem-solving perspectives were unique to each discipline (e.g., perseverance was emphasized in physics, empathy in nursing), while some perspectives were emphasized across several STEM fields (e.g., teamwork). Transferable problem-solving practices were often used alongside specialized practices to handle the unique challenges of each discipline. Findings suggest shifting away from framing problem-solving as a solitary transferable skill and toward framing problem-solving as an integrated set of context-dependent practices. (Supported by NSF DGE-1561493) 


\section{Introduction}

Problem-solving has been praised as a crucial skill for 21st-century workplaces; emphasized for its high transferability and its relevance across many disparate disciplines within STEM and beyond. [1] The development of problemsolving skills in undergraduate education is an essential topic in discipline-based education research, and especially within physics education research (PER) [2]. Furthermore, introductory physics courses have been heralded by instructors both in and out of physics as an ideal setting for undergraduates to develop problem-solving skills. These comments often frame problem-solving as a discrete isolated skill [3], something that can be cultivated in one situation and become immediately usable in multitudes of scenarios.

This transferability of problem-solving is an attractive concept $[1,4]$. Introductory physics courses are already required by many STEM degree programs, so integrating problemsolving skills is convenient. Additionally, if physics problemsolving was shown to be a transferable skill across many domains, degrees in physics would be seen as more valuable across disciplines; giving physics graduates greater flexibility of employment across many industries.

The goals of this study are to understand the breadth of problem-solving across several STEM fields and to understand how physics problem-solving fits within that spectrum. The uniqueness of physics problem-solving, particularly the mathematical nature, has long been a focus within physics education research [2]. But if physics has disciplinespecific problem-solving strategies, it stands to reason that other STEM disciplines could have unique problem-solving strategies as well.

We address the following research questions in our study

1. How do student perceptions of problem-solving vary across STEM disciplines and where are these perceptions formed?

2. How transferable is problem-solving as a skill from one STEM discipline to others?

3. How useful is the physics problem-solving learned in introductory physics classes for non-majors?

\section{Methodology}

This analysis was conducted as a sub-study of the larger EMPOWER project; a skills documentation initiative exploring communication, teamwork, problem-solving, and selfdirected learning in the 21st-century workplace to foster a more proficient and adaptable workforce and help inform educational practices. Undergraduates in nursing, energy, computing, manufacturing, and physics from two-year and fouryear institutions from four high-STEM metropolitan regions across the United States. Sixty-nine student focus groups were conducted, with two hundred thirty-nine students interviewed in total.

Interviews were transcribed, then coded in NVivo 11 and
12. On the first pass, three a priori schemes were applied to the transcripts: "Definition of Problem" for how an interviewee describes a problem they face (322 instances); "Strategies to Solve" for methods or characteristics of a problem solvers (618 instances); and "Teaching" for how problem solving is taught (730 instances). We applied these codes descriptively; meaning that preservation of student definitions and ideas about problem-solving were prioritized. Given that a major goal of this study is to elucidate variations in perceptions of problem-solving, instances where students describe "problem-solving" or "problems" were grouped together before the differences in these scenarios or acts were analyzed. The three a priori schemes were then open coded as a second pass (1324 codes).

After coding was complete, open codes were grouped by discipline. We used graphical software to create groupings for open codes that looked across disciplines. Groupings of codes were then arranged on a scale based on the relative frequency that they were mentioned across disciplines, with one end of the scale labeled "Multi-disciplinary/Transferable" and the other end labeled "Discipline-Specific/Specialized."

\section{Results}

Students from all fields asserted that solving problems is essential to working in their discipline. Often this was expressed as "Engineering is just problem-solving" or "Computing is just problem-solving," yet when these students elaborate as to what that phrase means to them, students from different disciplines would sometimes describe vastly different acts. Despite the ubiquity of problem-solving in STEM, it comes in various forms often dependent on disciplines. We present seven problem-solving aspects, ordered by the degree to which student emphasis was multi-disciplinary (e.g., collaborative problem-solving) or which only students in a single discipline emphasized (e.g., systems level thinking). There may be some surprises, such as perseverance (Sec. III A 7) being emphasized by only physics majors. This doesn't mean perseverance isn't relevant in other fields, just that students other fields emphasized other aspects of problem-solving instead. We now review each of the seven aspects.

\section{A. Discipline-Specificity of Student Problem-Solving}

\section{Collaborative Problem-Solving}

We defined collaborative problem-solving to be any problem-solving process which involves multiple people working together towards a common goal. By a large margin, collaborative problem-solving is the skill students describe most frequently across all industries. Modern workplaces naturally lend themselves to collaborative problem-solving as the problems faced are often too large or complicated to be solved by one person. As a result, collaborative problemsolving spans a vast range of separate problem-solving prac- 
tices, including but not limited to bouncing ideas off of each other, having each other's backs, communicating solutions to problems, give and take with your team, and diversity of experience. Many of these skills are highly transferable, allowing collaborative problem-solving to be effective across all the fields in our study.

"[...] I think that a lot of engineering is solving problems. And having different experiences is what helps you solve those problems in a team." - Manufacturing student, 2 year college

Working in teams is essential for solving problems. Teams can tackle much larger problems than individuals. Coordinating division of larger problems into parts that individuals can solve is a crucial team function, as the following student describes.

"[...] it's communicating the logic to your team because since most of these jobs that we're going into are team oriented it's- how are you writing this problem out, without using any code, just like writing it out and then explaining it to your team, how you're going to solve it because let's say you're the designer. The designer will create the overall problem to solve, and the developers will come in and code that out and create the functionalities" - Computing student, 2 year college

This student emphasizes not just the collaborative part to solving the problem, but also the importance of clarity of communication and roles.

\section{Information Seeking}

We defined information seeking as any form of searching for or gathering knowledge relevant to a given problem. Information seeking covers many various activities, from searching syntax on the internet, looking for symptoms, to question-asking. Given the complexity and uniqueness of many of problems across nursing, computing, energy, manufacturing, and physics, finding information needed to resolve a situation is seen across all the STEM fields studied. A core part of the problem-solving process is characterizing and learning about the problem itself. Information seeking often arose as an essential first-step in the problem-solving process. The following computing student describes seeking out information in the first steps of dealing with customer support,

“[...] you're probably dealing with someone that's not very technical trying to convey technical information. So you kind of have to weed through what they're telling you, kind of get the information that you need, and then be able to go and look and be like, 'Okay. So this is what we're seeing now. How do we start solving this problem?' " - Computing student, 2 year college

Oftentimes in IT and computing, problem-solving is medi- ated by clients who are not subject-matter experts. Information seeking is especially important in these contexts when the history of the problems are unknown. Similar difficulties are expressed by the following nursing student who discussed polypharmacy (when concurrent use of multiple medications by a patient changes outcomes).

"[...] when you're doing the assessment, you have to be able to ask questions, detailed questions, well questions well enough to know exactly what is going on with the patient, and I give an example. So, for instance, a patient is seeing like four different physicians. This patient can suffer from polypharmacy, so if you don't have any information, how do you know how to go from there." - Nursing student, 2 year college

This student describes as question-asking as an essential part of information gathering. Question-asking is helpful for clarifying goals and can inform where to look for information to help solve problems. [5].

\section{Handling Crises and Emotions}

We defined handling crises and emotions as being able to handle unexpected and high-stress situations while maintaining professionalism and focus. This is emphasized in disciplines where graduates will face high-stress problems with potentially fatal consequences if they are not solved appropriately. Nursing students mention handling crises and emotions most frequently, but a few examples were mentioned from students of other disciplines (e.g., IT students working for emergency call centers, energy students if pumps suddenly stop working on oil rigs). Students usually discuss this idea in terms of staying focused, emotional compartmentalization, empathizing with those in danger, working in fast-paced environments, and saving lives.

Nurses have a rigorous training for handling stressful situations. Examinations oftentimes stay true to authentic procedures, as the following student discusses.

"[...] you're on a time clock and you're being you're being video recorded and you know it's going to be shown in front of all your classmates and you're trying to remember CPR and it was just I learned so much in that lab because I had to think on my feet and the mistakes I made, the solutions to those are burned into me now, I will never forget them. I have a special part of my brain for how to reverse morphine is like burned in there, and it was really really good." - Nursing student, 4 year college

As this student describes, the solutions to some common problems are so vital to avoiding catastrophe that they need to be memorized completely. But outside of the classroom, crises can deeply affect people's lives. One student describes what it is like when working clinicals; the essential experiential learning for nursing students in an actual hospital environment. 
"I need to come and put everything that have been happening in my personal life aside and be able to really focus on my patients and have that empathy and have that compassion, but also be able to separate personal emotion so that I can think with a clear head, and think on my feet, and be free from error and so basically take it seriously every day." - Nursing student, 4 year college

Across disciplines which handle crises, students describe onthe-job crisis management as the balance this nursing student discusses - both understanding the importance of the situation and not letting the urgency become overwhelming.

\section{Problem Prevention}

Problem prevention emerged as a significant trend among students in the energy industry and among nursing students. We defined problem prevention as any steps taken to preserve an ideal state and to keep systems from deviating from a norm. As one nursing student describes problem prevention, "[...] the best way to solve a problem is to avoid it." - Nursing student, 4 year college.

This strategy of solving problems before they happen is the core of preventive problem-solving. Students mention many different practices contribute to problem prevention, such as automation, being free from error (mentioned previously), having an extra pair of eyes, and watching each others' backs. Ideally, deviations from the norm can be caught early and rectified, as this student describes,

"So problem solving, it's not necessarily just dealing with once you've already gotten an issue. It's being able to see how things are trending and being able to correct it before it turns into something that's actually a problem." - Energy student, 2 year college

A key distinction this student creates is between issues and things that are "actually a problem". Not all deviations from the norm are necessarily problems (e.g., patient thirstiness), but a collection of issues may point to a larger underlying problem. As described by students, problem prevention is resolving these smaller issues as they appear.

\section{Systems-Level Thinking}

Systems-level thinking is being able to conceptualize how all of the complex parts of a system interconnect and affect each other. Students studying for work in energy industries focus very strongly on system-level thinking. Working on oil rigs, a problem with a single component can affect multiple subsystems and interfaces, compounding the potential for errors. Awareness of the overall system is critical for safe and effective problem-solving. Students often discuss this in terms of seeing before and after a process, looking at how things are trending, conceptualizing everything going on, and knowing a process from start to finish.

"You have to know where everything is going because if you don't then you're not going to understand why this isn't working or what valve might be closed. I feel like all the diagrams will be kind of our platform to be able to troubleshoot everything." - Energy student, 2 year college

Being able to readily imagine every interaction of multiple interdependent systems is a daunting task, so the use of diagrams is essential for conceptualizing these systems. As a result, students studying these fields describe the many specialized measurement tools, visual aids, and monitoring systems used to understand the whole system as essential for troubleshooting and problem-solving.

\section{Adaptability}

We defined adaptability as the process of developing one's skills to match a problem over time or changing the framing of a problem to make it match one's own skill set. Although students from multiple disciplines mention adaptability in various contexts (e.g., adapting to a changing field), only physics majors mentioned adaptability as part of problemsolving. Physics majors usually discuss adaptability for problem-solving with phrases such as changing one's viewpoints or methods, changing how the problem appears, and handling problems across many different fields. Adaptability is not transferability as transferability is a quality of a skill while adapting is a process an individual undergoes to match different contexts or their own skills to a problem.[1]. The following physics student discusses how skills are adapted.

"[...] if you have some really tangled issue involving some mathematical simulation or something, you may need to draw on physics knowledge. You may need to draw on computation knowledge or algorithmic optimization or even computer hardware engineering. Could be something that you need to worry about, so being able to map between different domains and sort of see the end result and the path to it while solving a problem is really critical." - Physics major, 4 year college

Physical phenomena tend to be at the foundations of many other different domains, consequently students with physics knowledge may be more able to map problems across domains. Beyond this, the emphasis on model-based reasoning in physics may contribute to student competency at adapting models to fit the needs of the problem [6].

\section{Perseverance}

We defined perseverance as the ability to continue working on problems despite failure or hardship. Similar to adaptability, only physics students discussed perseverance in the context of problem-solving. When students of other 
disciplines talked about perseverance, discussions centered mostly on one's career path. Physics students often brought up strategies for problem-solving such as sticking with a problem, taking your time when solving, and patience with problems. Some students, including the following, mention explicitly that perseverance alone is not enough to solve problems, but that creativity must be combined so new solutions can be found.

"You're going to get stuck on things in the real world, stuck on problems and you have to be able to get-figure out how to work to get around them. You've got to stick with it because it's going to get frustrating, so you have to just be willing to try different things, basically." - Physics major, 4 year college

An important aspect is that trying different things does take time to do. For other disciplines, time pressure of the problems likely limits perseverance from becoming a useful tool for problem-solving (e.g. if a patient is in critical condition on a table, nurses do not have time to persevere through the problem for hours). Students in some other industries like computing and manufacturing mention failure as a part of an iterative explorative process of continuing to refine and improve new solutions for a single problem, while physics majors discussed perseverance in terms of grit and tenacity when solutions are not easily found.

\section{B. Integration of Problem-Solving Strategies}

Although some problem-solving strategies are used more generally while others are more discipline-specific, students describe most problem-solving acts diverse and multi-faceted processes. Even the most specialized problem-solving strategies were discussed alongside broader techniques, and what strategies students describe is very context dependent, often with multiple strategies used side-by-side. As one student describes,

"If you're working on a problem or something that you've been stuck on for anywhere from a week to two months, and someone comes in and tells you, 'Oh. It's actually wrong.' And they are correct. Being able to take that with a open mind would make it a lot easier to fix your problem and move on from there." - Physics student, 4 year college

This student concisely describes an important connection between Perseverance and Collaborative Problem-Solving. These strategies coexist, and much of the disciplinespecificity in problem-solving we found related to how each of the individual strategies interconnected.

\section{Solving "Real-World" Problems}

Students from every discipline expressed concepts of "realworld problems" which exist at the professional cores of each of their respective disciplines; what constitutes a real-world problem depends greatly on where students want to work in the future. These conceptions of real-world problems drastically influence students' perceptions of the utility and necessity of coursework, as this student describes

"I think our program here, specifically with [name], is super successful because he doesn't just teach SolidWorks, he teaches you how to use it in the real world." - Manufacturing student, 2 year college

Students often cite the incorporation of real-world problems in the classroom as something that increases engagement, and that lacking real-world problems can be a significant factor that can reduce engagement and enjoyment of a class.

\section{Conclusions}

The most significant finding is that there does not appear to be a single unified concept of problem-solving across disciplines. This is important for contexts where problem-solving is treated like a single isolated skill, such as job postings looking for recent graduates.

How students understand and implement problem-solving is linked to where and how they form their conceptions of problem-solving. Some contributions are outside of instructor influence, such as prior work experience before college or family connections to the industries they wish to pursue. Additionally, instructors with experience in a student's ideal industry may serve as essential models for students to see industry problems and problem-solving first-hand.

If introductory physics classrooms serve as forges for developing problem-solving skills across STEM, then instructors must participate with their students' ideas about realworld problem-solving. This upon consideration of the fact that many non-physics students taking introductory physics classes will eventually be employed within industries which face problems very different from rote physics problems. When appropriate for student goals, explicitly incorporating strategies such as collaborative problem-solving, handling crises and emotions, or information seeking may increase student engagement. The diversity of problem-solving across STEM is not just relevant when teaching classes for nonmajors, either. Physics graduates end up working in industries across STEM, so supporting physics majors' understanding of how problem-solving skills are specialized across industries will greatly aid in the process of adapting to the problems of their chosen workplace.

\section{Acknowledgments}

This work is supported by NSF Award DGE-1561493. The study design and data collection was done in conjunction with Matt Hora, Ross Benbow, Bailey Smolarek and other collaborators at the Wisconsin Center for Education Research. 
[1] National Research Council (U.S.), J. W. Pellegrino, and M. L. Hilton, eds., Education for life and work: developing transferable knowledge and skills in the 21st century (The National Academies Press, Washington, D.C, 2012).

[2] J. L. Docktorand J. P. Mestre, Synthesis of discipline-based education research in physics, Physical Review Special Topics - Physics Education Research 10, 10.1103/PhysRevSTPER.10.020119 (2014).

[3] N. S. Rebello, L. Cui, A. G. Bennett, D. A. Zollman, and D. J. Ozimek, Transfer of learning in problem solving in the context of mathematics and physics, Learning to solve complex scien- tific problems , 223 (2007).

[4] J. D. Bransfordand D. L. Schwartz, Chapter 3: Rethinking Transfer: A Simple Proposal With Multiple Implications, Review of Research in Education 24, 61 (1999).

[5] O. Eris, Asking generative design questions: a fundamental cognitive mechanism in design thinking (Center for Design Research, Stanford University, Stockholm, Sweden) p. 8.

[6] A. Pawl, A. Barrantes, D. E. Pritchard, M. Sabella, C. Henderson, and C. Singh, Modeling Applied to Problem Solving (MIT Press, Cambridge MA, Ann Arbor (MI), 2009) pp. 51-54. 